\title{
Le royaume du Bosphore Cimmérien aux époques grecque et romaine : Un aperçu
}

\section{Aleksandr Vasil'evič Podosinov}

Traducteur : Laurent Auberson

\section{OpenEdition}

\section{Journals}

Édition électronique

URL : http://journals.openedition.org/edl/328

DOI : $10.4000 /$ edl.328

ISSN : 2296-5084

\section{Éditeur}

Université de Lausanne

\section{Édition imprimée}

Date de publication : 15 mai 2012

Pagination : 87-110

ISBN : 978-2-940331-27-7

ISSN : 0014-2026

\section{Référence électronique}

Aleksandr Vasil'evič Podosinov, « Le royaume du Bosphore Cimmérien aux époques grecque et romaine : Un aperçu », Études de lettres [En ligne], 1-2 | 2012, mis en ligne le 15 mai 2015, consulté le 20 décembre 2020. URL : http://journals.openedition.org/edl/328 ; DOl : https://doi.org/10.4000/edl. 328 


\section{LE ROYAUME DU BOSPHORE CIMMÉRIEN AUX ÉPOQUES GRECQUE ET ROMAINE: UN APERÇU}

Le présent article propose un aperçu de l'histoire du royaume du Bosphore Cimmérien sur presque un millénaire, depuis le début du $\mathrm{V}^{\mathrm{e}}$ siècle av. J.-C. et jusqu’à l'invasion des Huns au $\mathrm{IV}^{\mathrm{e}}$ siècle de notre ère. On y analyse les processus d'avènement et de développement du royaume bosporan, une formation étatique unique dans l'histoire du monde antique, qui conciliait des éléments d'une organisation politique de type polis avec une forme de gouvernement monarchique. Ici, comme nulle part ailleurs, on peut observer les traits d'une synthèse gréco-barbare ethnopolitique et culturelle, qui se fit précédemment aux campagnes d'Alexandre le Grand. Tous les aspects de l'histoire du royaume bosporan sont examinés dans cette étude: politiques, socio-économiques, religieux, culturels, domestiques, etc.

Situé aux confins du monde grec habité, à la frontière avec la légendaire Scythie, le royaume du Bosphore Cimmérien est une réalité historique dont la durée s'étend sur près de mille ans, depuis la fondation des premières colonies grecques au VIe s. av. J.-C. jusqu'à l'invasion des Huns au IV s. apr. J.-C. ${ }^{1}$.

Dans cette histoire, il subsiste de nombreuses zones d'ombre, en particulier pour les premiers temps du royaume. Selon Diodore, les Archéanactides ('A $\chi \alpha \iota \alpha v \alpha \kappa \tau i \delta \alpha \imath)$ accédèrent au pouvoir vers $480 \mathrm{av}$.

I. Ce texte présente un bref aperçu de près de mille ans de l'histoire du Bosphore, telle qu'elle a été étudiée par de nombreuses générations d'historiens, d'archéologues, de philologues, d'épigraphistes et de numismates. Dans un cadre aussi restreint, il n'était pas possible de produire des références pour toutes les propositions soutenues ici. Le lecteur trouvera en fin d'article une liste des principaux ouvrages traitant de l'histoire du Bosphore Cimmérien, de même qu'une chronologie de ses souverains. 
J.-C. et gouvernèrent pendant quarante-deux ans ${ }^{2}$. On ne connaît précisément ni l'origine des Archéanactides, ni les circonstances qui les ont amenés au pouvoir, ni la manière dont ils l'ont exercé, ni enfin les raisons qui ont contraint des cités jusqu'alors indépendantes à se regrouper en un Etat. Une hypothèse rattache les Archéanactides à une famille aristocratique des premiers colons venus de Milet ou de Mytilène, où est attesté le nom d'Archaianax.

Les Archéanactides, de même que les Spartocides plus tard, exerçaient vraisemblablement leur pouvoir au titre d'archontes, ce qui préservait du moins les apparences d'une constitution démocratique. Mais la nature héréditaire de ce pouvoir, en désaccord avec les traditions de la démocratie grecque, favorisa le passage d'une organisation politique de type polis à une monarchie. Panticapée, la plus grande des cités grecques, avantageusement située, fut choisie pour capitale de ce nouveau royaume.

Pour expliquer un tel regroupement de cités naguère autonomes, les historiens invoquent généralement, en plus de raisons économiques, la nécessité de se défendre contre un danger extérieur. Or les Scythes se faisaient de plus en plus menaçants et l'on suppose que de nombreuses cités du Bosphore qui avaient pu s'en passer jusqu'alors se mirent à construire des fortifications. L'imposante muraille de Tiritaka, qui relie la ville à la mer d'Azov, au nord, sur un tracé de quelque vingt-cinq kilomètres, fut construite sous les Archéanactides pour défendre la frontière occidentale du royaume contre les nomades scythes.

Vers le milieu ou dans la deuxième moitié du $\mathrm{V}^{\mathrm{e}}$ s. av. J.-C., un temple dédié à Apollon fut construit à Panticapée. C'est aussi à cette époque que commence la frappe des monnaies à la légende АПО $\Lambda$. Ces faits permettent de supposer avec une certaine vraisemblance que les cités du Bosphore s'étaient regroupées en une amphictyonie, c'est-à-dire une alliance de nature à la fois religieuse et politique.

En 438 av. J.-C., toujours selon Diodore ${ }^{3}$, une nouvelle dynastie, celle des Spartocides, établit sa domination sur le Bosphore, pour plus de trois cents ans, jusqu'à la fin du $\mathrm{II}^{\mathrm{e}}$ s. av. J.-C. On ignore malheureusement les circonstances qui amenèrent ce changement. Ce qui est certain toutefois, c'est que le nom du fondateur de la nouvelle dynastie - Spartokos - et celui de plusieurs de ses successeurs sont thraces. Beaucoup d'historiens

2. Bibliothèque historique XII.31.1.

3. Ibid. 
supposent par conséquent que le pouvoir parvint ainsi aux mains de représentants d'une noblesse locale scytho-thrace, mais déjà fortement hellénisée.

Quoique l'on ignore pratiquement tout du fondateur de la dynastie, Spartokos I ${ }^{\mathrm{er}}$, qui resta sept ans au pouvoir (438/437-433/432), on est en droit de supposer qu'il fut le premier artisan des énergiques travaux de fortification et d'extension du territoire de l'Etat qui, à cette époque, comprenait déjà plusieurs cités et colonies grecques sur les deux rives du détroit de Kertch (Panticapée, Porthmion, Myrmèkion, Tiritaka, Acre, Kytaia et Kimmèrikon sur la rive occidentale, Phanagorie, Kèpoi, Hermonassa, Tyramba, Patraios, Achilleion et d'autres encore sur la rive orientale). Sont considérées comme des cités Panticapée, Phanagorie, Hermonassa et Kèpoi, de même que les villes annexées plus tard au royaume du Bosphore, Nymphaion, Théodosie, Gorgippia (Port des Sindes) et Tanaïs, fondée ultérieurement.

Cité grecque jusqu'alors indépendante, Nymphaion, fondée au début du VI ${ }^{e}$ siècle par des colons ioniens, fut la première victime de la politique expansionniste des Spartocides. Nymphaion entretenait des relations particulières avec Athènes, et il est possible qu'après l'expédition pontique de Périclès, elle fit partie de la Ligue de Délos et fut placée sous la juridiction d'Athènes qui, très certainement, voulait s'assurer le contrôle du commerce du blé du Bosphore. Quelques sources littéraires et épigraphiques laissent supposer que Nymphaion versait à la Ligue de Délos un tribut qui d'un talent passa en 425 à deux talents.

Après Nymphaion, ce fut au tour de Théodosie d'être annexée au royaume, au terme d'un long combat. Colonie milésienne fondée dans la deuxième moitié du $\mathrm{VI}^{\mathrm{e}}$ siècle, Théodosie faisait apparemment concurrence au royaume du Bosphore dans le commerce du blé. Théodosie, le seul port de cette partie de la Crimée non exposé au gel, fut prise sous le règne de Leukôn I ${ }^{\text {er }}$, probablement dans les années 380 .

Après avoir soumis Théodosie, les Spartocides s'intitulèrent (précisément à partir du règne de Leukôn) «archôn du Bosphore et de

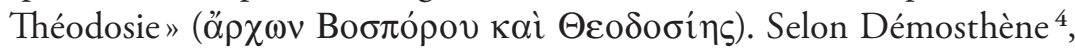
Leukôn fit construire à Théodosie un port de commerce dont la qualité n’avait rien à envier à celui de Panticapée.

4. Contre Leptine 33. 
Au début du $\mathrm{IV}^{\mathrm{e}}$ siècle av. J.-C., pour protéger leur pays contre les incursions des habitants des steppes de Crimée, les souverains du Bosphore firent édifier ou réparer un système de fortifications réparties sur une ligne nord-sud à l'ouest des villes de la côte (par exemple le fossé d'Uzunlar).

Sous le règne des premiers Spartocides, Satyros I ${ }^{\text {er }}$ et Leukôn I ${ }^{\text {er }}$, le royaume s'étendit aussi vers l'est. L'objectif était maintenant d'obtenir la soumission de la presqu'île de Taman et de la côte orientale de la mer d'Azov, qui était habitée par des tribus sindo-méotes. La Sindikè, déjà fortement hellénisée depuis le $\mathrm{V}^{\mathrm{e}}$ siècle par ses liens étroits avec le Bosphore, fut la première visée. Son annexion ne fut réalisée qu’après la soumission de Théodosie, ainsi que nous l'apprend une inscription récemment découverte au kourgane des Sept-Frères (en Sindikè).

Ce fut ensuite le tour d'autres tribus, dont nous pouvons suivre l'incorporation progressive au royaume du Bosphore à l'évolution de la titulature des souverains. Leukôn fut le premier à s'intituler «archôn du Bosphore et de Théodosie, roi des Sindes, des Torètes, des Dandariens

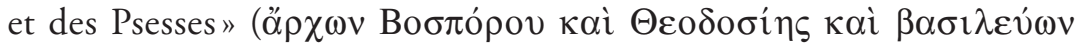

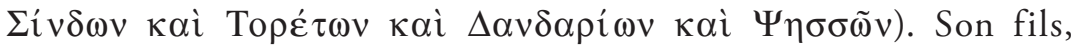

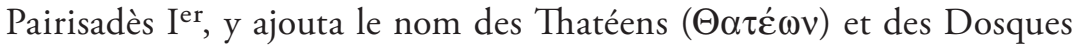
$(\Delta o ́ \sigma \chi \omega v)$, quand il ne se nommait pas simplement «roi de tous les

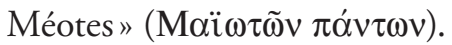

Dans le troisième quart du IV e siècle av. J.-C., la formation territoriale du royaume du Bosphore était pour l'essentiel achevée. Avec ses $5000 \mathrm{~km}^{2}$, ce territoire était le plus grand Etat grec de l'époque après la Sicile dionysienne. Il comprenait une trentaine de villes et de grandes colonies, de statuts divers, et un vaste arrière-pays agricole. Son extension n’allait pratiquement plus changer jusqu'à la fin de l'Antiquité.

Il ne fait aucun doute que l'incorporation d'un "pays barbare» aussi étendu exerça une influence sur la genèse du modèle politique développé plus tard par les Etats hellénistiques. Ce "phénomène bosporan" a parfois aussi été considéré comme une forme de «Protohellénisme" 5 . Pour la question de l'évolution du pouvoir monarchique dans le Bosphore, il est en tout cas significatif qu'à partir du III e siècle av. J.-C., les

5. Il faut comprendre le terme d' "Hellénisme» comme correspondant à la période hellénistique, selon l'acception que lui en a donné J. G. Droysen (1808-1884). Le "Protohellénisme» caractérise donc la période immédiatement antérieure (nde). 
Spartocides (et déjà Spartokos III) se donnèrent le titre de «rois" de tous les sujets du royaume du Bosphore, alors que leurs prédécesseurs, au $\mathrm{V}^{\mathrm{e}}$ et au IV siècle, se qualifiaient encore d' "archontes" des villes grecques et de « rois» des tribus barbares.

Pour qualifier la forme de gouvernement des souverains du Bosphore, on peut utiliser sans hésiter le terme de tyrannie. C'est généralement du nom de tyrans qu'ils sont désignés par les auteurs antiques, et occasionnellement de dynastes ou de rois.

De nombreuses inscriptions du $\mathrm{V}^{\mathrm{e}}$ et du $\mathrm{IV}^{\mathrm{e}}$ siècle nous apprennent que le tyran du Bosphore exerçait le pouvoir avec son frère ou ses fils, ce qui donne un caractère collégial au régime. Pour les historiens modernes, l'institution du pouvoir collégial est même une caractéristique de l'Etat bosporan.

Le pouvoir ne s'appuyait pas sur une milice populaire de citoyens, mais sur une armée de mercenaires et sur les troupes scythes alliées. Il en allait donc tout autrement que dans les démocraties grecques classiques, mais ce système contribua à la longévité du régime tyrannique.

Le royaume ne put connaître une telle prospérité et son commerce une telle expansion dans le monde grec que grâce à une stabilité politique, sociale et économique qui reposait sur l'équilibre entre les deux piliers du pouvoir spartocide, à savoir les habitants grecs des cités et les Barbares indigènes.

Des orateurs attiques comme Lysias, Isocrate et Démosthène, et des inscriptions attiques ou bosporanes nous renseignent sur les quantités impressionnantes de blé livrées à Athènes au IV ${ }^{\mathrm{e}}$ siècle. Selon Démosthène $^{6}$, dans la seule année 356 , les Spartocides, qui détenaient le monopole de ce commerce, fournirent aux Athéniens 400000 médimnes de blé (soit 12400 tonnes), le tout franc de taxes. Le prix qu'Athènes devait payer pour une telle quantité de blé importé peut être estimé à 333 talents. Pour le transport, il fallait environ 150 bateaux. Un décret honorifique, inscription découverte sur l'acropole d'Athènes ${ }^{7}$, nous apprend qu'en 284, le roi Spartokos III (fils d'Eumélos, qui régna de 304/303 à 284/283) fit cadeau aux Athéniens de 15000 médimnes (environ 465 tonnes) de blé. Les destinataires reconnaissants lui firent

6. Contre Leptine 31-32.

7. $I G \mathrm{II}^{2}, 653$. 
élever sur l'agora et sur l'acropole d'Athènes deux statues de bronze «à côté des statues de ses ancêtres".

Le Bosphore exportait d'autres marchandises aussi, comme du poisson, des peaux et de la laine, et importait du vin, de l'huile d'olive, des produits artisanaux et des articles de luxe. Le vin provenait de Chios, de Thasos, de Cnide, de Cos, de Paros et d'Héraclée du Pont, l'huile d'Attique et de Sinope, et de l'Attique également les articles de luxe, les vases et la vaisselle ${ }^{8}$.

Le Bosphore vit aussi se développer un artisanat indigène, notamment la céramique (vaisselle et tuiles), la toreutique et le tissage, toutes activités dont l'existence est bien attestée par l'archéologie.

Les souverains du Bosphore sont aussi connus pour avoir entretenu une flotte commerciale et militaire. Leukôn et ses fils firent construire dans la partie orientale du port de Panticapée des docks qui pouvaient contenir une trentaine de bâtiments et servaient à la fois de bassins de radoub et de chantier naval ${ }^{9}$. Ils disposaient d'une armée de quatre mille mercenaires.

Fondée sur le commerce de céréales, la prospérité du Bosphore trouve son reflet dans le monnayage des souverains: Leukôn fit frapper les premières monnaies d'or, qui avaient même cours en dehors du royaume. Les poinçons monétaires révèlent le très haut degré de maîtrise atteint par les graveurs.

Parmi les Spartocides, Leukôn I ${ }^{\text {er }}$, dont le règne dura quarante ans, acquit une grande notoriété dans le monde antique. Ayant su réunir des cités grecques en un Etat, ayant soumis de vastes régions barbares aux alentours, ayant bâti une puissance économique grâce aux exportations de blé vers Athènes, et tissé des relations avec de nombreux Etats de Grèce et du pourtour de la mer Noire, Leukôn et son royaume jouissaient d'une renommée qui s'étendait dans tout le monde grec, au point que les auteurs antiques firent souvent de Leukôn le fondateur de la dynastie des Spartocides, aussi appelés Leukonides ${ }^{10}$.

Nous ne connaissons pas dans le détail la vie et le règne de tous les successeurs de Leukôn; de plusieurs, nous n'avons que des mentions dans

\footnotetext{
8. Cf. Polybe IV.38.4-5.

9. Cf. Strabon VII.4.4.

Io. Cf. par exemple Elien, Histoire variée VI.13.
} 
les sources littéraires, épigraphiques ou numismatiques. Pour quelquesuns, la documentation est plus abondante.

Par un des décrets honorifiques athéniens dont il a déjà été question, de l'année 347/346, nous sommes quelque peu renseignés sur Pairisadès $I^{\mathrm{er}}$, fils de Leukôn, qui partagea le pouvoir avec son frère Spartokos II. On suppose aussi qu'il élargit les acquisitions territoriales de son père dans la partie asiatique du Bosphore ou qu'il les rétablit après une révolte, puisque sa titulature le désigne comme «roi des Sindes, de tous les Méotes et des Thatéens». Selon Diodore, son fils Eumélos s'acquit un titre de gloire en menant la guerre «contre les peuples barbares qui s'adonnaient à la piraterie, les Hénioques, les Taures et les Achéens" et en "débarrassant la mer des pirates qui y sévissaient». Il "fit passer sous sa domination une bonne partie des régions environnantes habitées par les Barbares et accrut le lustre de son royaume. Il avait entrepris de soumettre tous les peuples entourant le Pont-Euxin, et il y serait rapidement parvenu si sa vie n'avait pris fin si brutalement" ${ }^{11}$.

Des symptômes d'une crise économique et politique se manifestèrent dès le III ${ }^{\mathrm{e}}$ siècle av. J.-C. Les émissions monétaires et les refrappes sont révélatrices de problèmes financiers: les types des monnaies de cuivre se dégradèrent et changèrent souvent, les frappes de monnaies d'argent et d'or cessèrent. Les difficultés économiques - la chose est remarquable - eurent également des incidences sur les offrandes funéraires, qui devinrent plus modestes.

Cette époque coïncide avec un regain d'activité des peuples barbares des contrées au nord de la mer Noire: dans les steppes, les Sarmates consolidèrent leur hégémonie, tandis qu'en Crimée se constituait un royaume scythe. A la fin du II siècle, la dynastie des Spartocides succomba à cette pression et le royaume du Bosphore perdit sa souveraineté.

Ainsi que les historiens l'ont déjà fait souvent observer, le voisinage des peuples barbares explique pour une part non négligeable le regroupement précoce des cités du Bosphore sous l'autorité d'un Etat central.

Le mouvement se fit dans les deux sens. D’une part l'élément barbare pénétra profondément dans le milieu grec des cités, et d'autre part, les Barbares s'hellénisèrent de plus en plus, comme le montrent les témoignages de l'archéologie, en particulier les nécropoles des cités grecques, et ceux de l'onomastique et des sources littéraires.

II. Bibliothèque historique XX.25.3. 
Plusieurs tribus, qui reconnaissaient l'autorité du roi du Bosphore, s'établirent en sédentaires dans le royaume. Par un processus intensif d'acculturation, ils furent intégrés à la vie des cités grecques et formèrent bientôt une partie de la population des villes et des villages, donnant ainsi naissance à un Etat gréco-barbare du type de celui que connaissaient les régimes despotiques de l'Orient hellénistique.

Les kourganes bosporans, particulièrement fréquents au $\mathrm{IV}^{\mathrm{e}}$ et au III ${ }^{\mathrm{e}}$ siècle av. J.-C., sont des exemples caractéristiques des interactions et de l'acculturation gréco-barbare. Certains de ces tumuli présentent en effet des rites funéraires typiquement grecs, et quelques-uns, de toute évidence, abritaient même la tombe d'un roi du Bosphore. C'est le cas par exemple du "Kourgane Royal» découvert près de Panticapée, tombeau probable de Leukôn $I^{\text {er }}$ ou de Pairisadès I ${ }^{\text {er }}$. D'autres kourganes (comme celui de Koul-Oba, à l'ouest de Panticapée) ont servi de sépulture aux représentants d'une aristocratie locale fortement hellénisée. Pour les périodes ultérieures également, les archéologues distinguent entre les rites funéraires grecs, barbares ou mixtes.

La ville de Tanaïs, fondée au III' siècle à l'embouchure du fleuve du même nom (le Don actuel), à un emplacement d'importance stratégique pour le commerce, est le meilleur exemple de symbiose gréco-barbare (synœecisme). De par sa situation géographique et son rôle d'avant-poste des échanges avec l'arrière-pays barbare de la steppe au nord-est de la mer d'Azov, Tanaïs fut dès le début une colonie mixte. Comme le notait Strabon:

Tanaïs [...] servait de marché d'échange à la fois pour les nomades d'Asie et d'Europe et pour les navigateurs venus du Bosphore trafiquer sur le Méotide, les premiers procurant des esclaves, des peaux et en général ce que peuvent offrir des nomades, les seconds apportant en contrepartie des vêtements, du vin et tout ce qui est propre à la vie civilisée ${ }^{12}$.

Dans les années 220-200, Tanaïs était devenue une cité grecque munie de toutes les institutions propres à ce statut. A la fin du III ${ }^{\mathrm{e}}$ siècle, une importante colonie peuplée de Barbares se forma dans le faubourg de la ville. Tanaïs était ainsi divisée en deux parties, l'une barbare (les Tanaïtes) et l'autre grecque (les Hellènes), division qui se retrouvait

I2. Strabon XI.2.3. Trad. François Lasserre, Paris, Les Belles-Lettres, 1975. 
dans l'organisation des magistratures municipales: plusieurs inscriptions mentionnent en effet deux charges distinctes, celle d'hellênar-

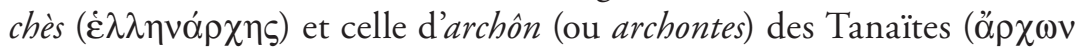

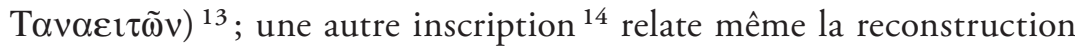

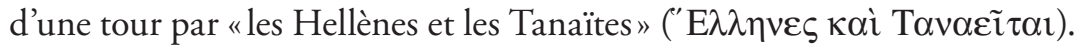
Nombreux sont les éléments de la culture barbare qui passèrent dans la vie des Tanaïtes, comme l'usage de la céramique modelée ou les rites funéraires, qui font apparaître un mélange de traditions grecques et barbares (sarmates).

Parmi les articles "propres à la vie civilisée» que les Grecs fournissaient aux Barbares, les objets d'orfèvrerie, tels que bijoux féminins, vases, peignes ou gorytes ${ }^{15}$, tiennent une place éminente. Les objets de ce genre se rencontrent fréquemment dans les tombes des Scythes, des Sarmates ou d'autres peuples barbares et ils forment une part essentielle de ce que l'on appelle "l'or des Scythes", qui remplit les cabinets d'orfèvrerie des grands musées de Russie et d'Ukraine. La plupart des chercheurs sont d'accord pour attribuer la fabrication de ces objets précieux, dont beaucoup imitent le style animalier des Scythes, à des ateliers du Bosphore Cimmérien ou d'autres villes du littoral septentrional du Pont-Euxin.

Les villes du Bosphore avaient une vie culturelle animée. Nous n'en donnerons que deux exemples. Sur un sarcophage de pierre du $I^{\text {er }}$ siècle apr. J.-C., trouvé à Kertch et maintenant conservé au Musée de l'Ermitage à Saint-Pétersbourg, est représenté l'atelier d'un peintre, lequel se tient assis avec un pinceau devant son chevalet. Aux murs sont accrochés des tableaux montrant des figures humaines: il s'agit donc d'un peintre de portraits. Et à Kertch également, a été découvert un caveau funéraire, dont les murs sont ornés de riches peintures représentant la tête de Déméter et l'enlèvement de Coré par Hadès.

Il y avait aussi dans ces cités des théâtres et des gymnases. Des poètes composaient des vers pour les inscriptions dédicatoires et funéraires. Pour son passage sur les rivalités entre les fils de Pairisadès ${ }^{\text {er }}$, Diodore a utilisé le texte d'un historien local dont le nom ne nous est pas connu. Sphaïros du Bosphore, philosophe grec célèbre, fut le conseiller du roi

I3. $C I R B 1242,1245,1251$ a.

I4. CIRB 1243.

I5. NdT: garnitures métalliques pour étuis à arc. 
de Sparte Cléomène. Et à l'époque romaine, il se donnait des jeux de gladiateurs à Panticapée.

L'univers religieux des Bosporans était semblable à celui du reste du monde grec. Panticapée honorait avant tout Apollon Médecin, divinité tutélaire à laquelle elle avait dédié le temple principal sur l'acropole. La large diffusion du culte de Déméter et de celui de Dionysos s'explique par l'importance de l'agriculture dans le royaume du Bosphore. On connaît à Panticapée des temples dédiés à Artémis, Aphrodite, Asclépios, Déméter, Arès, Héraclès, parmi d'autres divinités du panthéon hellénique. De nombreuses cités du Bosphore furent également perméables aux cultes de Cybèle, Mithra, Attis, Sérapis, Isis et d'autres dieux encore. Les habitants se regroupaient souvent en associations ou confréries religieuses (synodos, thiasos).

Des inscriptions attestent dans plusieurs villes la présence d'une importante communauté juive, dont les dédicaces étaient adressées au "Dieu suprême" (theos hypsistos). La communauté chrétienne, apparue plus tard, eut des évêques qui participèrent au concile de Nicée en 325 puis aux autres conciles œcuméniques.

Le règne de Mithridate VI Eupator, roi du Pont (121/120-63 av. J.-C.), marque le début d'une nouvelle ère pour le royaume du Bosphore. Dans sa lutte contre Rome, Mithridate avait en effet besoin de regrouper toutes les ressources politiques, économiques et militaires des pays riverains de la mer Noire. Lorsqu'à la fin du II e siècle, la ville de Chersonèse, menacée par les Scythes de Crimée, lui demanda de l'aide, il n'hésita pas longtemps à saisir l'occasion unique qui lui était ainsi offerte. En 110, un corps expéditionnaire fut envoyé à Chersonèse sous le commandement du général Diophante. Ces évènements sont relatés dans une inscription honorifique que lui dédièrent les Chersonésites. Diophante y est célébré comme un vainqueur qui anéantit les armées du roi scythe Palakos et s'empara des principales citadelles de la Crimée scythe, Néapolis des Scythes et Chabaioi. La campagne de Diophante eut pour le royaume du Bosphore des conséquences importantes sur lesquelles nous renseigne aussi ce même décret honorifique:

Après s'être rendu dans le territoire du Bosphore, Diophante régla les affaires locales au mieux et selon les intérêts du roi Mithridate Eupator; puis lorsque les Scythes, sous la conduite de Saumakos, eurent déclenché un soulèvement, tué le roi du Bosphore Pairisadès, qui l'avait élevé 
[Saumakos], et monté une conjuration contre Diophante, celui-ci réussit à échapper au danger en montant sur un bateau que les citoyens lui avaient apprêté, vint [vers nous], harcela les citoyens de ses prières et obtint l'appui empressé de son célèbre roi Mithridate Eupator; au début du printemps, il parut avec une armée de terre et une marine de guerre, fit monter les guerriers choisis par les citoyens sur trois bateaux, quitta notre ville, conquit Théodosie et Panticapée, punit les insurgés, s'empara de Saumakos, le meurtrier du roi Pairisadès, et l'envoya dans le royaume [de Mithridate], rétablissant ainsi la puissance du roi Mithridate ${ }^{16}$.

L'inscription laisse entendre que le roi du Bosphore Pairisadès V, avant la révolte scythe, remit de son plein gré à Mithridate la souveraineté sur le Bosphore. Les historiens modernes situent la révolte de Saumakos en 107 av. J.-C. Peut-être Saumakos faisait-il partie de la maison royale des Scythes de Crimée et, après la défaite de Palakos devant Chersonèse, avait-il réussi à s'emparer de la capitale du Bosphore. Mais il est possible aussi qu'il se soit trouvé parmi les mercenaires scythes au service de Pairisadès et que, profitant de la faiblesse du roi et de la situation politico-militaire du moment, il renversa le dernier Spartocide et usurpa son pouvoir. Quoi qu'il en soit, le Bosphore fut désormais intégré au royaume du Pont.

C'est finalement dans le Bosphore Cimmérien qu'allaient se dérouler les évènements tragiques liés à l'effondrement du royaume de Mithridate et à la défaite du roi dans sa lutte contre Rome, et c'est là qu'il allait mourir.

Sur les derniers jours de Mithridate, Appien nous a laissé une relation détaillée ${ }^{17}$. Battu par Pompée et trahi par son fils Macharès, Mithridate avait fui dans les régions septentrionales de la mer Noire, où il avait à nouveau soumis à son autorité le Bosphore Cimmérien et Chersonèse. Il tenta une seconde fois de rassembler des forces armées et des moyens afin de poursuivre la guerre contre Rome, mais les cités du Bosphore, épuisées par ce long conflit, se soulevèrent les unes après les autres. Enfermé dans la forteresse de l'acropole de Panticapée, assiégé, Mithridate fut finalement contraint au suicide. La même année (63 av. J.-C.), une grande partie de la ville de Panticapée fut détruite par un séisme.

I6. IosPE, $\mathrm{I}^{2}, 352$.

I7. Mithridatique 101-111. 
Après la défaite de Mithridate, le Bosphore Cimmérien entra dans la sphère d'influence de Rome. Pharnace, qui avait joué un rôle décisif dans la chute de son père, fut reconnu roi du Bosphore et reçut le titre d'«ami

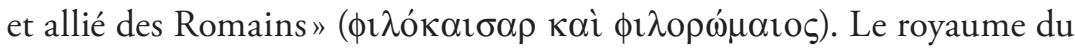
Bosphore acquit ainsi le statut d'Etat vassal de Rome.

Désireux de conquérir une partie des anciennes possessions de Mithridate, Pharnace se jeta sur l'Asie Mineure, mais fut vaincu par César à la bataille de Zéla en 47 av. J.-C. (cette guerre éclair fut à l'origine de la célèbre formule: veni, vidi, vici), et dut se replier dans le Bosphore. Il fut tué par Asandros, qu'il avait lui-même institué comme gouverneur. Asandros régna ensuite trente ans sur le Bosphore.

Par défiance envers Asandros, Rome envoya Mithridate de Pergame, fils illégitime de Mithridate Eupator, dans le Bosphore pour s'y faire reconnaître comme roi. Mais il ne réussit pas à destituer Asandros et fut lui-même tué. Après la mort de César, Asandros, qui avait d'abord porté le simple titre d'archôn, se revêtit de celui de roi, qui lui fut plus tard reconnu par Rome.

Afin de conférer à son pouvoir la légitimité dont il avait besoin, Asandros épousa une petite-fille de Mithridate Eupator, nommée Dynamis, qui allait reprendre les rênes de l'Etat après la mort de son mari en 17 av. J.-C. Mais peu après déjà, un certain Scribonius éleva des prétentions au trône du Bosphore. Rome tenta à nouveau de profiter de l'occasion et d'exploiter les troubles à son avantage en envoyant dans le Bosphore son protégé, le roi du Pont Polémon. Celui-ci essaya d'une part de légitimer ses prétentions au trône en épousant Dynamis et d'autre part d'incorporer officiellement à son royaume du Pont les territoires ainsi nouvellement acquis. Mais la mort violente qu'il trouva en 8 av. J.-C. dans un combat contre des tribus locales mit un terme à ses projets. Dynamis eut de nouveau en mains la destinée politique du Bosphore Cimmérien, jusqu'à la prise du pouvoir par son fils Aspourgos en 10 apr. J.-C.

Aspourgos fut le fondateur d'une dynastie qui allait régner presque quatre siècles sur le royaume du Bosphore. Il prit le titre de roi en 14 apr. J.-C., avec l'accord de l'empereur Tibère, et régna jusqu'à sa mort en 38. A cette époque, le Bosphore entretenait des relations très changeantes avec Rome, qui intervenait régulièrement dans la succession au trône du Bosphore.

Néron envisagea de faire du Bosphore Cimmérien une province romaine. Lagitation chronique des Scythes de Crimée (Tauroscythes) 
lui fournit le prétexte pour organiser en 63 une grande expédition militaire contre la Crimée, sous la conduite du légat de la province de Mésie, Tiberius Plautius Silvanus Aelianus. De nombreuses garnisons furent installées, abritant chacune environ trois mille hommes en armes. Une quarantaine de vaisseaux de guerre patrouillaient sur la mer Noire. Plusieurs inscriptions funéraires nous autorisent à supposer que des détachements romains furent également stationnés sur le territoire du royaume du Bosphore.

La suite de l'histoire du Bosphore Cimmérien se caractérise par les guerres permanentes que les rois durent mener contre divers peuples barbares, tels les Tauroscythes, les Sarmates, les Siraces, les Alains, etc. La réaction particulièrement énergique de Sauromatès II (174-210) fut couronnée de succès, puisqu'il réussit à pacifier ou à soumettre les Scythes de Crimée. Un succès éphémère cependant, et le ciel se faisait de plus en plus menaçant au-dessus du royaume du Bosphore. Les témoignages archéologiques et épigraphiques montrent qu'au cours du $\mathrm{II}^{\mathrm{e}}$ et du III siècle, aux confins orientaux du royaume et sur la rive nord de la mer d'Azov, de nombreuses citadelles bosporanes furent abandonnées et de nouvelles fortifications construites à la hâte.

C'est une époque où les souverains du Bosphore aimaient à se parer de somptueux titres à l'orientale, comme "Roi des Rois" ( $\beta \alpha \sigma \imath \lambda \varepsilon v ́ \varsigma$ $\beta \alpha \sigma \imath \lambda \dot{\varepsilon} \omega v)$ ou "Grand Roi » ( $\mu \dot{\varepsilon} \gamma \alpha \varsigma \beta \alpha \sigma \imath \lambda \varepsilon v \varsigma \varsigma)$. Ils faisaient frapper leur effigie sur les monnaies du royaume, parfois sous les traits de souverains divinisés. Leurs noms révèlent par ailleurs de plus en plus des origines iraniennes (Thothorsès, Rhadamsadios) ou thraces (Cotys, Rhescouporis). Quatre rois portèrent le nom de Sauromatès.

Le royaume du Bosphore, pas plus que les autres Etats du littoral nord de la mer Noire, ne fut épargné par la crise qui gagna le monde antique au III ${ }^{\mathrm{e}}$ siècle. Les symptômes en sont à la fois politiques, militaires, économiques et sociaux. Les affranchissements d'esclaves furent nombreux, à en croire les témoignages épigraphiques, tandis que l'on assistait à un retour à l'économie naturelle, à une régression de l'activité commerciale et à un recul de la culture urbaine.

La menace la plus grave, pour le royaume du Bosphore, fut celle des vagues successives d'invasions déclenchées par les grandes migrations de peuples barbares. Au début du III ${ }^{\mathrm{e}}$ siècle, les Goths, venus des côtes de la Baltique, envahirent le littoral nord de la mer Noire. Dans la basse vallée du Danube, ils s'engagèrent dans une longue lutte avec Rome; 
en même temps, poussant vers l'est, ils détruisirent Olbia et pénétrèrent dans le territoire du Bosphore avec d'autres peuples, les uns germaniques et les autres iraniens. Gorgippia succomba dans les années 230 sous les assauts de tribus barbares (probablement des Alains), et vers 250, Tanaïs subit un sort semblable. Dans les années 250-270, des bandes de Goths, d'Hérules, de Boranes et de Greuthunges se livrèrent à des expéditions de piraterie contre des villes du nord de la mer Noire, de la Propontide et de l'Egée, poussant même jusqu'à Athènes, en prenant comme bases les ports et comme moyen de transport les bateaux du Bosphore Cimmérien.

Les incursions des Goths eurent de graves conséquences pour le Bosphore. Des destructions et des signes de déclin sont perceptibles dans beaucoup de villes et de villages à cette époque. Mais au début du $\mathrm{IV}^{\mathrm{e}}$ siècle, le royaume existait toujours.

Signe sûr de son effondrement économique, dans les années 330340 , sous le règne de Rhescouporis IV, le royaume, après neuf siècles de frappe presque sans interruption, mit fin à son activité monétaire.

Ce furent les Huns qui donnèrent le coup de grâce au royaume du Bosphore. Arrivés depuis peu d'Asie et nomadisant entre la Volga et le Don, ils franchirent en 375 le Tanaïs (Don), c'est-à-dire la frontière de l'Europe, anéantirent le royaume goth de Hermanarich, puis le royaume ostrogoth d'Atanarich et parvinrent dans la basse vallée du Danube. Une de leurs hordes, venant de l'est, dans la région de la mer d'Azov, mit à sac la presqu'île de Taman, franchit le Bosphore Cimmérien et pilla encore la partie européenne du royaume. L'invasion des Huns mit fin à l'histoire millénaire de l'antique royaume du Bosphore.

Panticapée et quelques autres villes du royaume se remirent cependant. Dans l'Empire byzantin dont elle fit partie dès la fin du $\mathrm{V}^{\mathrm{e}}$ siècle ou le début du VI $\mathrm{VI}^{\mathrm{e}}$ siècle, Panticapée joua encore un rôle non négligeable. Hermonassa apparaît à maintes reprises dans des sources médiévales, sous le nom de Tamatarcha (et plus tard Tmutarakan'), et les témoignages archéologiques sur la vie dans les villes et les villages ne manquent pas. Le christianisme s'implanta au $\mathrm{V}^{\mathrm{e}}$ siècle et le Bosphore constitua même un diocèse. Il est donc légitime de parler malgré tout d'une continuité entre l'Antiquité et le Moyen Age.

L'histoire du royaume du Bosphore, pour conclure, révèle dans la constitution politique, l'économie et la culture de nombreux traits particuliers qui tiennent aux relations de voisinage entre les colonies 
grecques et les populations indigènes, nomades ou sédentaires, du littoral nord de la mer Noire. C'est ce qui fait l'intérêt de cette région pour la connaissance des sociétés antiques.

Aleksandr Vasil'evič Podosınov

Université d'Etat des Sciences Humaines, Moscou

Traduction de l'allemand de Laurent Auberson 


\section{ANNEXE I}

Liste des quelques ouvrages de référence sur l'histoire du Bosphore Cimmérien

Alekseeva, Ekaterina Mikhajlovna, Antičnyj gorod Gorgippija [La ville antique de Gorgippia], Moskva, Editorial URSS, 1997.

Anokhin, Vladilen Afanas'evič, Monetnoe delo Bospora [Le monnayage du Bosphore], Kiev, Naukova Dumka, 1986.

—, Istorija Bospora Kimmerijskogo [Histoire du Bosphore Cimmérien], Kiev, Odigitrija, 1999.

Blavatskaja, Tat'jana Vasil'evna, Očerki političeskoj istorii Bospora v $V-I V v v$. do n. è. [Essais sur l'histoire politique du Bosphore aux $V^{e}-I V^{e}$ siècles avant notre ère], Moskva, Izdatel'stvo Akademii Nauk SSSR, 1959.

Blavatskij, Vladimir Dmitrievič, Pantikapej [Panticapée], Moskva, Nauka, 1964.

Bolgov, Nikolaj Nikolaevič, Zakat antičnogo Bospora: Očerki istorii Bosporskogo gosudarstva pozdneantičnogo vremeni (IV-V vv.) [Le crépuscule du Bosphore antique: essais sur l'histoire de l'Etat bosporan d'époque tardive (IV $V^{e}-V^{e}$ siècles)], Belgorod, Izdatel'stvo Belgorodskogo Gosudarstvennogo Pedagogičeskogo Universiteta, 1996.

Braund, David (ed.), Scythians and Greeks: cultural interactions in Scythia, Athens and the early Roman Empire (six century BC - first century $A D$ ), Exeter, University of Exeter Press, 2005.

$C I R B=$ STRUVE, Vasilij Vasil'evič et alii, Korpus bosporskikh nadpisej [Corpus des inscriptions bosporanes], Moskva/Leningrad, Nauka, 1965.

Fornasier, Jochen, Böttger, Burkhard, Das Bosporanische Reich: der Nordosten des Schwarzen Meeres in der Antike, Mainz, Philipp von Zabern, 2002.

Frolova, Nina Andreevna, The Coinage of the Kingdom of Bosporus A.D. 69-238, Oxford, BAR International, 1979. 
-, The Coinage of the Kingdom of Bosporus A.D. 242-341/342, Oxford, BAR International, 1983.

Gajdukevič, Viktor Francevič, Das Bosporanische Reich, Berlin/ Amsterdam, 1971.

Graham, Alexander John, "Thasos and the Bosporan Kingdom", Ancient West \& East, 1.1 (2002), p. 87-101.

Heinen, Heinz, Antike am Rande der Steppe. Der nördliche Schwarzmeerraum als Forschungsaufgabe, Mainz/Stuttgart, Franz Steiner Verlag, 2006.

Hind, John G. F., "The Bosporan Kingdom", in Cambridge ancient history $\mathrm{VI}^{2}$ : The fourth century B.C., Cambridge, Cambridge University Press, 1994, p. 476-511.

$I G=$ Inscriptiones Graecae, Berlin, 1983-.

IosPE = Inscriptiones orae septentrionalisPonti Euxini Graecae et latinae, ed. V. Latyšev, T. 1, 2, 4, Petropoli, 1885-1901.

Kallistov, Dmitrij Pavlovič, Očerki po istorii severnogo Pričernomor'ja antičnoj epokhi [Essais sur l'histoire du littoral septentrional de la mer Noire à l'époque antique], Leningrad, Izdatel'stvo Leningradskogo Gosudarstvennogo Universiteta, 1949.

Kobylina, Marija Mikhajlovna, Fanagorija, Moscou, 1956 (MIA 57).

Kruglikova, Irina Timofeevna, Bospor v pozdneantičnoe vremja [Le Bosphore durant l'Antiquité tardive], Moskva, Nauka, 1966.

Kuznecov, Vladimir Dmitrievič, Košelenko, Gennadij Andreevič, Očerki arkheologii $i$ istorii Bospora [Essais sur l'archéologie et l'histoire du Bosphore], Moskva, Nauka, 1992.

Maslennikov, Aleksandr Aleksandrovič, Naselenie Bosporskogo gosudarstva v VI-II vv. do n.è. [La population de l'Etat bosporan aux $V I^{e}-I I^{e}$ siècles avant notre ère], Moskva, 1981.

Molev, Evgenij Aleksandrovič, Bospor v period ellinizma [Le Bosphore durant la période hellénistique], Nižnij Novgorod, Izdatel'stvo Nižegorodskogo Gosudarstvennogo Universiteta, 1994.

—, Političeskaja istorija Bospora v VI-IV vv. do n. è. [Histoire politique du Bosphore aux VIe-IVe siècles avant notre ère], Nižnij Novgorod, Izdatel'stvo Nižegorodskogo Gosudarstvennogo Universiteta, 1997.

Moreno, Alfonso, Feeding the democracy: The Athenian grain supply in the fifth and fourth centuries $B C$, Oxford, Oxford University Press, 2007. 
Müller, Christel, D'Olbia à Tanaïs: territoires et réseaux d'échanges dans la Mer Noire septentrionale aux époques classique et hellénistique, Paris, De Boccard, 2010.

Podossinov, Alexandr Vasil'evič, "Am Rande der griechischen Oikumene: Geschichte des Bosporanischen Reiches", in Das Bosporanische Reich: der Nordosten des Schwarzen Meeres in der Antike, hrsg. von Jochen Fornasier und Burkhard Böttger, Mainz, Philipp von Zabern, 2002, p. 21-38.

Rostowzew, M[ikhail Ivanovič], Skythien und der Bosporus, Bd. I: Kritische Übersicht der schriftlichen und archäologishen Quellen, Berlin, H. Schoetz, 1931 (erweiterte Fassung der russischen Ausgabe von 1925).

-, Skythien und der Bosporus, Bd. II: Wiederentdeckte Kapitel und Verwandtes, übersetzt und herausgegeben von H. Heinen, Stuttgart, Philipp von Zabern, 1993.

Saprykin, Sergej Jur'evič, Pontijskoe tsarstvo [Le royaume du Pont], Moskva, Nauka, 1996.

—, Bosporskoe tsarstvo na rubeže dvukh epokh [Le royaume du Bosphore à la charnière de deux époques], Moskva, Nauka, 2002.

ŠElov, Dmitrij Borisovič, Monetnoe delo Bospora v VI-II vv. do.n.è. [Le monnayage du Bosphore aux VIe-IIe siècles avant notre ère], Moskva, Izdatel'stvo Akademii Nauk SSSR, 1956.

Šelov-Kovedjaev, Fjodor Vadimovič, "Istorija Bospora v VI-IV vv. do n. è.» ["Histoire du Bosphore aux $\mathrm{VI}^{\mathrm{e}}$-IV ${ }^{\mathrm{e}}$ siècles avant notre ère»], in Drevnejšie gosudarstva na territorii SSSR [Les Etats les plus anciens sur le territoire de l'URSS], Moskva, 1985, p. 5-187 (Materialy i issledovanija 1984).

Tolstikov, Vladimir Petrovič, «K probleme obrazovanija Bosporskogo gosudarstva (opyt rekonstrukcii voenno-političeskoj situacii na Bospore v konce VI - pervoj polovine V v. do n. è.» [ «Le problème de la formation de l'Etat bosporan (essai de reconstitution de la situation militaro-politique au Bosphore à la fin du VI ${ }^{\mathrm{e}}$ siècle et durant la première moitié du $\mathrm{V}^{\mathrm{e}}$ siècle avant notre ère»], Vestnik Drevnej Istorii, 3 (1984), p. 24-48.

-, "Descriptions of Fortifications of the Classical Cities in the Region to the North of the Black Sea", Ancient Civilizations from Scythia to Siberia, 4.3 (1998), p. 188-231. 
Tsvetaeva, Galina Aleksandrovna, Bospor i Rim [Le Bosphore et Rome], Moscou, Nauka, 1979.

Ustinova, Yulia, The supreme Gods of the Bosporan Kingdom: celestial Aphrodite and the most high God, Leiden, E. J. Brill, 1999.

Vinogradov, Jurij Germanovič, "Die historische Entwicklung der Poleis des nördlichen Schwarzmeergebiets im 5. Jh. v. Chr.", Chiron, 10 (1980), p. 63-100.

Werner, Robert, «Die Dynastie der Spartokiden», Historia, 4 (1955), p. $412-444$.

-, «Die griechische Schwarzmeerkolonisation und das Bosporanische Reich ", in Handbuch der Geschichte Russlands, Bd. 1.1, hrsg. von Manfred Hellmann, Stuttgart, Anton Hiersemann, 1981, p. $153-172$.

Zavojkin, Aleksej Andreevič, Obrazovanie Bosporskogo gosudarstva. Arkheologija i khronologija stanovlenija territorial'noj deržavy [ La formation de l'Etat bosporan. Archéologie et chronologie évolutive d'une puissance territoriale], Moscou, 2007 (dissertation inédite).

Žebeljov, Sergej Aleksandrovič, Severnoe Pričernomor'e [Le littoral septentrional de la mer Noire], Moscou/Leningrad, Izdatel'stvo Akademii Nauk SSSR, 1953. 


\section{ANNEXE II}

Chronologie des souverains du Bosphore

\begin{tabular}{|c|c|}
\hline 480-438 av. J.-C. & Archéanactides \\
\hline $438 / 437-433 / 432$ & Spartokos Ier \\
\hline $433 / 432-393 / 392$ & Satyros I $\mathrm{I}^{\mathrm{er}}$ (règne conjoint avec Séleukos I ${ }^{\mathrm{er}}$ ) \\
\hline $393 / 392-389 / 388$ & Satyros I Ir (règne seul) \\
\hline $389 / 388-349 / 348$ & Leukôn I ${ }^{\text {er }}$ \\
\hline $349 / 348-344 / 343$ & Spartokos II (règne conjoint avec Pairisadès Ier) \\
\hline $344 / 343-311 / 310$ & Pairisadès I $\mathrm{I}^{\mathrm{r}}$ \\
\hline $311 / 310-310 / 309$ & Satyros II (règne conjoint avec Prytanis?) \\
\hline $310 / 309$ & Prytanis Ir (cf. Diodore XX.22-24) \\
\hline $310 / 309-304 / 303$ & Eumélos \\
\hline $304 / 303-284 / 283$ & $\begin{array}{l}\text { Spartokos III (en partie règne conjoint avec } \\
\text { Séleukos II) }\end{array}$ \\
\hline $284 / 283$-env. 245 & $\begin{array}{l}\text { Pairisadès II (en partie règne conjoint avec } \\
\text { Satyros II) }\end{array}$ \\
\hline env. $245-240$ & Spartokos IV \\
\hline env. $240-220$ & Leukôn II \\
\hline env. $220-200$ & Hygiainon \\
\hline env. $200-180$ & Spartokos V \\
\hline env. $180-160$ & $\begin{array}{l}\text { Kamasarye (règne seul au début, puis avec } \\
\text { Pairisadès III et Pairisadès IV) }\end{array}$ \\
\hline env. $180-170$ & Pairisadès III (règne conjoint avec Kamasarye) \\
\hline env. $170-150$ & $\begin{array}{l}\text { Pairisadès IV Philometor (au début règne conjoint } \\
\text { avec Kamasarye, puis seul?) }\end{array}$ \\
\hline env. $150-140$ & Spartokos VI (?) \\
\hline env. 140-109 & Pairisadès V \\
\hline 108-107 (?) & Saumakos \\
\hline $107-63$ & Mithridate VI Eupator \\
\hline
\end{tabular}




\begin{tabular}{|l|l|}
\hline $63-47$ & Pharnace \\
\hline $47-17$ & Asandros \\
\hline $17 / 16$ & Dynamis \\
\hline 15 (?) & Scribonius \\
\hline $14-9 / 8$ & Polémon \\
\hline 9/8 av. J.-C.-7/8 apr. J.-C. & Dynamis \\
\hline $7 / 8-9 / 10$ apr. J.-C. & inconnu \\
\hline $10 / 11-37 / 38$ & Aspourgos (= Rhescouporis Ir ?) \\
\hline $37 / 38-38 / 39$ & Gepaipyris (veuve d'Aspourgos) \\
\hline $39 / 40-44 / 45$ (?) & Mithridate VIII \\
\hline $45 / 46-67 / 68$ & Cotys I'r (peut-être avec des droits limités de 62 \\
\hline à 67) \\
\hline $98-92 / 93$ & Rhescouporis II \\
\hline $123 / 124-132 / 133$ & Sauromatès I'r \\
\hline $131 / 132-153 / 154$ & Cotys II \\
\hline $153 / 154-170 / 171$ (173?) & Rhoimétalkès \\
\hline $173 / 174-210 / 211$ & Tib. Iulios Eupator \\
\hline $210 / 211-226 / 227$ & Sauromatès II \\
\hline $227 / 228-233 / 234$ & Rhescouporis III \\
\hline $229 / 230-231 / 232$ & Cotys III \\
\hline $233 / 234$ & Sauromatès III \\
\hline $234 / 235-238$ & Rhescouporis IV \\
\hline $239 / 240-275 / 276$ & Ininthimeus \\
\hline $253 / 254$ & Rhescouporis V \\
\hline 266 & Pharsanzès \\
\hline $275 / 276$ & Chedosbios \\
\hline $275 / 276-278 / 279$ & Sauromatès IV \\
\hline $278 / 279-285 / 286$ (?) & Tib. Iulios Teiranès \\
\hline $286 / 287-308 / 309$ & Chedosbios \\
\hline $308 / 309-322$ & Thothorsès \\
\hline $314-319$ et 322 & Rhadamsadios \\
\hline $323-342$ & règne conjoint avec Rhescouporis VI \\
\hline & Rhescouporis VI (règne peut-être plus long) \\
\hline
\end{tabular}




\section{ANNEXE III}

Les illustrations qui composent l'annexe III se trouvent aux planches VI à XV. L'éditeur est redevable au Musée d'Etat de l'Ermitage à SaintPétersbourg, et en particulier à Mme N. Ju. Novosyolova, de lui avoir fourni nombre des illustrations de cette annexe.

\section{Crédits iconographiques}

Pl. VI:

Musée d'Etat de l'Ermitage, $\mathrm{N}^{\circ}$ inv. R.1. (Vladimir Terebenin, Leonard Kheifets, Yuri Molodkovets (C The State Hermitage Museum, St. Petersburg).

Pl. VII :

Musée d'Etat de l'Ermitage, No inv. PAN-140. (Vladimir Terebenin, Leonard Kheifets, Yuri Molodkovets (C) The State Hermitage Museum, St. Petersburg).

Pl. VIII:

Infographie de Laurent Saget (C) UNIL/IASA, d'après A. A. Maslennikov, Drevnie zemljanye pogranično-oboronitel'nye sooruženija Vostočnogo Kryma [Ouvrages défensifs antiques en terre de Crimée orientale], Moskva, Institut arkheologii RAN, 2003, p. 26, fig. a.

Pl. IX.1 :

Musée d'Etat de l'Ermitage, No inv. P.1834.110. (Vladimir Terebenin, Leonard Kheifets, Yuri Molodkovets (C) The State Hermitage Museum, St. Petersburg). 
Pl. IX.2 :

Musée d'Etat de l'Ermitage, $N^{\circ}$ inv. NO-A3-65/N630. (Vladimir Terebenin, Leonard Kheifets, Yuri Molodkovets (C) The State Hermitage Museum, St. Petersburg).

Pl. X:

Photographie de Pascal Burgunder (C) UNIL/IASA.

Pl. XI:

Musée d'Etat de l'Ermitage, $N^{\circ}$ inv. KO 11. (Vladimir Terebenin, Leonard Kheifets, Yuri Molodkovets (C) The State Hermitage Museum, St. Petersburg).

Pl. XII :

Musée d'Etat de l'Ermitage, No inv. P.1899.81. (Natalia Antonova, Inna Regentova (C) The State Hermitage Museum, St. Petersburg).

Pl. XIII :

Reconstitution de Vladimir Tolstikov/Musée des Beaux-Arts Pouchkine, tirée de M. Koromila, The Greeks and the Black Sea from the Bronze Age to the Early Twentieth Century, Athènes, Panorama, 2002, fig. 173.

Pl. XIV :

Musée d'Etat de l'Ermitage, No inv. FA-1869.7. (Vladimir Terebenin, Leonard Kheifets, Yuri Molodkovets (C) The State Hermitage Museum, St. Petersburg).

Pl. XV:

Musée d'Etat de l'Ermitage, No inv. PAN-1726. a et b. (Vladimir Terebenin, Leonard Kheifets, Yuri Molodkovets (C) The State Hermitage Museum, St. Petersburg). 
